\title{
Sialoadenite esclerosante crônica (tumor de Kuttner): relato de caso clínico
}

\section{Chronic sclerosing sialadenitis (Kuttner tumour): clinical case report}

Rhayany de Castro Lindenblatt'; Janaína Barbieri dos Santos'; Damião Roballo Alves²; Simone de Queiroz Chaves Lourenço3; Eliane Pedra Dias ${ }^{4}$

\section{unitermos resumo}

Sialoadenite esclerosante

crônica

Tumor de Kuttner

Glândula submandibular
A sialoadenite esclerosante (tumor de Kuttner) representa um processo inflamatório crônico incomum de glândula salivar, que acomete com maior freqüência a glândula submandibular. O objetivo deste trabalho é relatar o caso clínico de um paciente que apresentou sialoadenite esclerosante crônica associada a quadro de sialolitíase. Essa entidade patológica é pouco freqüente, clinicamente pode ser confundida com uma neoplasia e, embora seja resolvida com a exérese da lesão, causa transtornos importantes ao paciente, como dor intensa, disfonia e disfagia. abstract

Chronic sclerosing sialadenitis (Kuttner tumour) is an unusual chronic inflammatory process of the salivary gland that affects principally the submandibular gland. The aim of this work is to report a case of chronic sclerosing sialadenits associated with sialolithiasis. Kuttner tumour is an uncommon entity that clinically may not be distinguished from neoplasia and though it can be resolved by surgical excision of the lesion, it causes important upset for the patient, as intense pain, dysphonia and dysphagia. key words

Chronic sclerosing

sialoadenitis

Kuttner tumour

Submandibular gland

\section{Introdução}

O tumor de Kuttner (TK), também denominado sialoadenite esclerosante crônica (SEC), representa um processo inflamatório crônico fibrosante incomum de glândula salivar, que acomete quase exclusivamente a glândula submandibular e freqüentemente pode não ser distinguido de uma neoplasia verdadeira ${ }^{(8)}$.

Foi primeiramente descrito na literatura germânica por Kuttner, em 1896, sendo reconhecido como entidade distinta pela Organização Mundial de Saúde (OMS) em $1991^{(9)}$. Em virtude de sua semelhança com uma neoplasia verdadeira e da falta de casos reportados na literatura, tornou-se uma lesão pouco conhecida por clínicos e patologistas ${ }^{(1)}$.

Acomete adultos, em ampla faixa etária (13 a 81 anos), sem predileção por sexo, com ocorrência predominantemente unilateral e grau de envolvimento glandular variável. Os aspectos clínicos do TK caracterizam-se por aumento de volume difuso, de consistência endurecida, assintomático, na região glandular, semelhante a um crescimento neoplásico ${ }^{(1-3,7,8)}$.

1. Mestrandas em Patologia Bucodental na Universidade Federal Fluminense (UFF).

2. Cirurgião bucomaxilofacial; curso de especialização em Estomatologia na Odontoclínica Central do Exército (OCEx/Rl).

3. Professora adjunta do Departamento de Patologia da UFF.

4. Professora titular do Departamento de Patologia da UFF.

Trabalho realizado no Programa de Pós-Graduação em Patologia do Departamento de Patologia da UfF e apresentado no XIV Congresso Brasileiro de Estomatologia (2006), sob a forma de apresentação oral de caso clínico. 
A associação dessa entidade com sialolitíase pode ser vista em $30 \%$ a $83 \%$ dos casos. Sabe-se que desordens da secreção glandular, inflamação linfocitária e reações imunes também podem se relacionar com sua etiopatogênese $\mathrm{e}^{(1,7)}$.

Microscopicamente, classifica-se a SEC em quatro estágios de acordo com seu grau de inflamação e fibrose. O estágio 1 caracteriza-se por um infiltrado inflamatório linfocitário focal, periductal. 0 estágio 2 mostra infiltração difusa de linfócitos, atrofia acinar acompanhada por fibrose periductal e lobular. Observa-se, também, metaplasia com proliferação do epitélio ductal. No estágio 3 ocorre redução do parênquima secretor da glândula, desenvolvimento de folículos linfóides secundários, com centros germinativos reativos e proliferação de ductos, além da ocorrência de metaplasia escamosa. O estágio 4 mostra destruição da arquitetura tubular, com perda do parênquima glandular e esclerose severa ${ }^{(1,2)}$.

A duração dos sintomas antes do tratamento é variável, de meses a décadas, e depende do tamanho da lesão. A hipótese diagnóstica em geral é neoplasia de glândula salivar(1).

A SEC deve ser diagnosticada através da realização do exame histopatológico, já que sua histopatologia está bem estabelecida na literatura. O prognóstico é favorável, e não existem relatos de transformação maligna ${ }^{(7)}$.

\section{Relato do caso}

Paciente do sexo masculino, negro, 24 anos, apresentou-se em consulta ao setor de emergência bucomaxilofacial do Hospital Regional de Araruama (RJ), com a seguinte queixa principal: "inchaço embaixo do queixo e dentro da boca".

Durante a anamnese, não foram relatadas doenças sistêmicas relevantes. Ao exame extrabucal, observou-se tumefação em região submandibular esquerda, sensível à palpação, com evolução de seis anos. Foi também relatada sintomatologia dolorosa durante alimentação, deglutição e fonação, além de xerostomia. No exame intrabucal, notou-se a presença de um aumento de volume nodular, de consistência endurecida, base séssil, superfície lisa e hipocrômica, dolorosa à palpação, sobrejacente ao ducto de Wharton, em assoalho bucal.

Ao exame de radiografias extra-orais lateral e oblíqua de mandíbula, foi observada imagem radiopaca triangular bem delimitada, de contornos irregulares, medindo 3,8 $\mathrm{x}$
2,2 x 1,6 cm em seus maiores diâmetros, localizada próximo ao ângulo mandibular esquerdo, estendendo-se dos terços apicais dos molares esquerdos à região submandibular do mesmo lado, superpondo-se ao osso hióide (Figura 1).

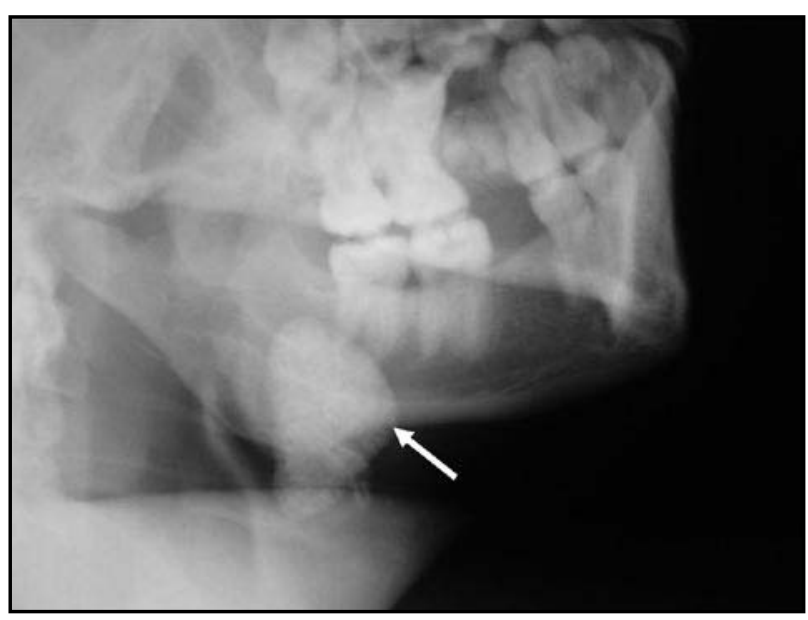

Figura 1 - Radiografia inicial

As hipóteses diagnósticas foram sialoadenite, sialolitíase e neoplasia de glândula salivar. Foi realizada excisão cirúrgica da lesão, com remoção total da glândula submandibular esquerda, por acesso extra-oral, sob anestesia geral. Foi obtido, além do tecido mole referente à glândula, um sialolito medindo, $3 \times 2 \times 1,6 \mathrm{~cm}$.

Todo o material foi encaminhado ao Serviço de Anatomia Patológica do Hospital Universitário Antônio Pedro (HUAP). Ao exame microscópico, observou-se intenso infiltrado inflamatório linfocitário, destruição dos ácinos (Figura 2), fibrose periductal e ectasia dos ductos (Figura 3). Áreas de metaplasia do epitélio ductal foram evidenciadas. A destruição do parênquima secretor glandular por intensa fibrose também foi encontrada em toda extensão do corte (Figura 4).

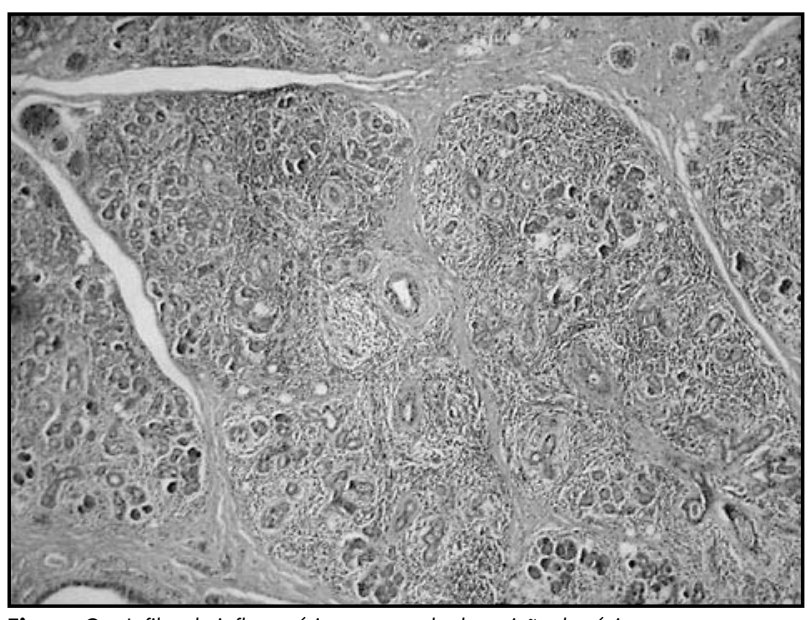

Figura 2 - Infiltrado inflamatório provocando destruição dos ácinos 


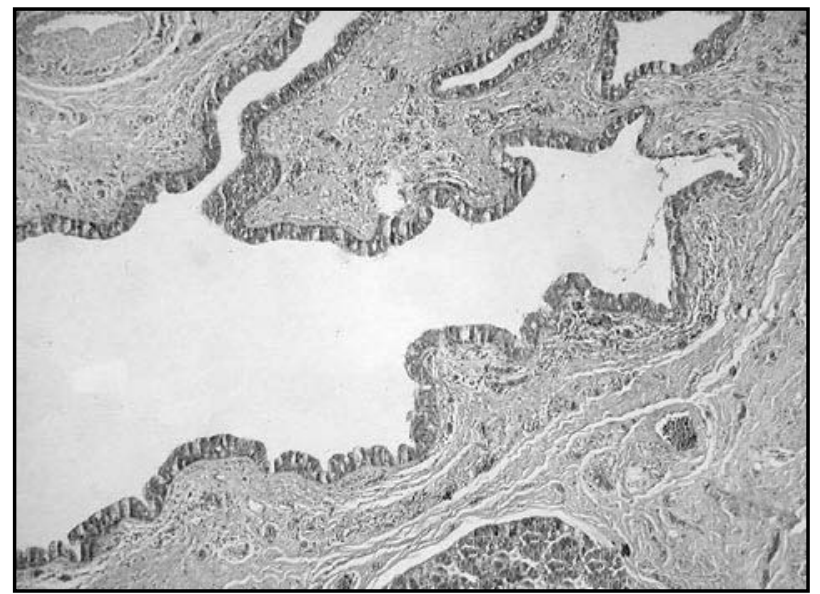

Figura 3 - Ectasia ductal e fibrose periductal

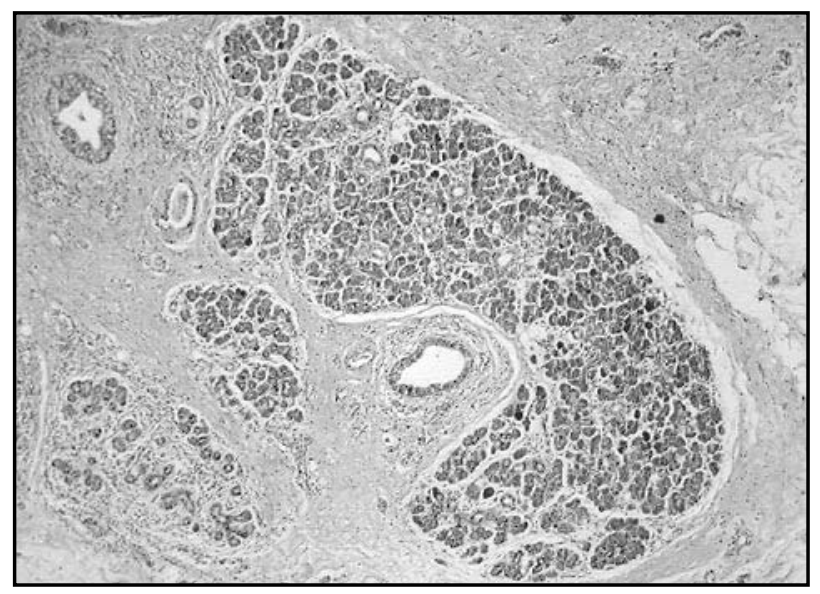

Figura 4 - Substituição do parênquima glandular por intensa fibrose

O diagnóstico definitivo foi de sialoadenite esclerosante crônica (Tumor de Kuttner) associada a sialolitíase. Em proservação após o tratamento cirúrgico (Figura 5) não foram observadas alterações. O paciente permanece em acompanhamento semestral, não apresentando mudanças até a presente data.

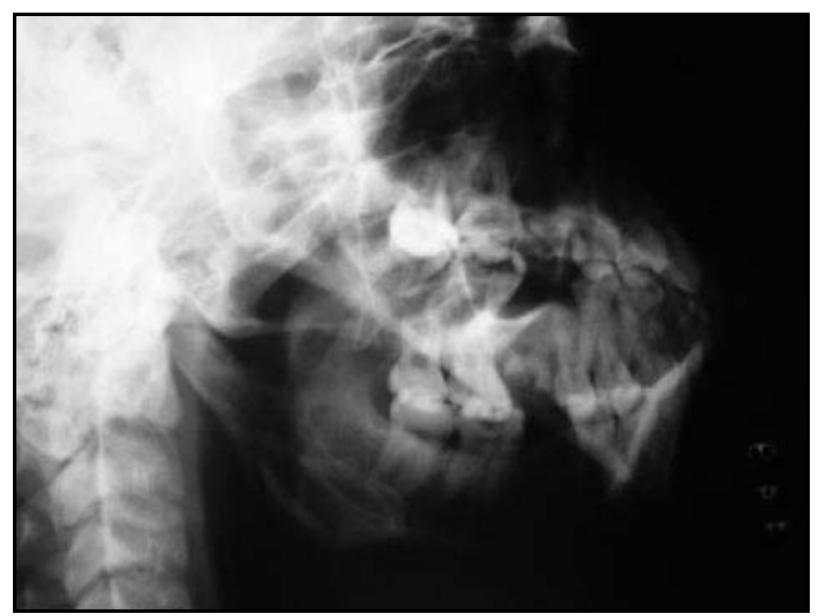

Figura 5 - Proservação após tratamento cirúrgico: não foram observadas alterações (6 meses)

\section{Discussão}

O TK representa uma condição inflamatória incomum que afeta as glândulas salivares maiores e clinicamente apresenta-se como um aumento de volume de consistência endurecida, com importantes dimensões, o que dificulta sua distinção de uma neoplasia verdadeira. Entretanto, o diagnóstico histopatológico não se torna difícil quando o patologista tem conhecimento de sua existência ${ }^{(1,4,8)}$.

Embora tenha suas etiologia, fisiopatologia e evolução descritas há muito na literatura, essa lesão constitui uma entidade pouco conhecida por clínicos e patologistas, em virtude do pequeno número de casos publicados até os dias de hoje $\mathrm{e}^{(1,4)}$.

O caso relatado neste trabalho refere-se a um paciente masculino, negro, com 24 anos de idade, que buscava tratamento da lesão há seis anos. Há relatos em que a faixa etária varia dos 12 aos 83 anos de idade, o que torna procedente a idade do paciente em questão. Existem dúvidas em relação à prevalência do gênero, pois de acordo com a literatura o sexo masculino é o mais acometido, enquanto Harrison et al. ${ }^{(6)}$ relatam a prevalência do feminino ${ }^{(1,4)}$.

Em razão de sua apresentação clínica o TK mimetiza uma neoplasia. Suas características histopatológicas propiciam o diagnóstico diferencial com tumores fibroblásticos, linfoma esclerosante, neoplasias de glândulas salivares, lesões linfoepiteliais benignas, doença de Kimura, linfoma extranodal marginal de células $B$ e tumor pseudo-inflamatório, o que torna relevante o conhecimento de seus aspectos histopatológicos para obtenção de um diagnóstico preciso ${ }^{(1,9)}$.

O presente caso difere das lesões linfoepiteliais benignas por não apresentar ilhas mioepiteliais. Além disso, não foi observada presença de infiltrado inflamatório eosinofílico, característica presente na doença de Kimura. A preservação da arquitetura glandular e a uniformidade celular desfavorecem os diagnósticos de linfoma extranodal marginal de células $B$, assim como o de pseudotumor inflamatório(1,9).

No caso apresentado observa-se ao exame histopatológico a presença de todos os estágios, predominando o estágio 3, compatível com o tempo de evolução da lesão, o que está de acordo com os achados da literatura ${ }^{(1,9)}$.

A sialolitíase é uma alteração comum das glândulas salivares e a maior causa de disfunção glandular(5). Autores como Huang et al. (2002) $)^{(7)}$, Adachi et al. (2004) $)^{(1)}$ e Ahuja et al. (2003) $)^{(2)}$ relatam que a associação com sialolitos pode estar presente em $30 \%$ a $83 \%$ dos casos e que esses cálculos geralmente estão localizados na porção terminal do sistema ductal, o que diverge deste caso, uma vez que o sialolito localiza-se na porção glandular do ducto. 
Apesar disso, ainda existem dúvidas sobre o sialolito ser causa ou conseqüência do TK. Hung et al. $(2002)^{(7)} \mathrm{e}$ Adachi et al. (2004)(1) alegaram que um sialolito ou qualquer obstrução gera inflamação e infecção crônicas que, somadas ao acúmulo de células inflamatórias, podem levar a edema dos ácinos glandulares, necrose, ectasia ductal e retenção de saliva, acompanhados de edema e inflamação, o que caracteriza uma sialoadenite esclerosante. Outros autores afirmam ser o sialolito secundário ao processo inflamatório, desencadeando o estabelecimento desse tumor ${ }^{(1,7)}$

\section{Considerações finais}

A SEC representa uma entidade clinicopatológica distinta, reconhecida pela OMS em 1991, que consiste em lesão inflamatória benigna incomum, semelhante a uma neoplasia verdadeira. Torna-se necessário o conhecimento de suas características para o estabelecimento do diagnóstico preciso, bem como do tratamento imediato e adequado, compatíveis com uma entidade benigna que pode comprometer as funções de fala, alimentação e deglutição do paciente.

\section{Referências}

1. ADACHI, M. et al. A case of Kuttner tumor of the submandibular gland. Auris Nasus Larynx, v. 31, p. 309-12, 2004.

2. AHUJA, A. T. et al. Kuttner tumour (chronic sclerosing sialadenitis) of the submandibular gland: sonographic appearances. Ultrasound Med Biol, v. 29, n. 7, p. 913-9, 2003.

3. BIALEK, E. J. et al. US-appearance of a Küttner tumour resembling a malignant lesion: US-histopatologic correlation. Eur J Ultrasound, v. 14, p. 167-70, 2001.

4. BLANCO, M. et al. Chronic sclerosing sialadenitis (Kuttner tumor): unusual presentation with bilateral involvement of major minor salivary glands. Ann Diagn Pathol, v. 7 , n. 1, p. 25-30, 2003.

5. BODNER, L.; BEER-SHEVA, I. Giant salivary gland calculi: diagnostic imaging and surgical management. Oral Surg Oral Med Oral Pathol, v. 94, n. 3, p. 320-3, 2002.
6. HARRISON, J. D. et al. Role of microliths in the etiology of chronic submandibular sialadenitis: a clinicopathological investigation of 154 cases. Histopathology, v. 31, p. 237-51, 1997.

7. HUANG, C. et al. Kuttner tumor chronic sclerosing sialadenitis. Am J Otolaryngol, v. 23, n. 6, p. 394-7, 2002.

8. LARBCHAROENUB, N.; PONGTIPPAN, A. Bilateral Kuttner tumor of submandibular glands: a case report and review of the literature. J Med Assoc Thai, v. 88, n. 3, p. 430-4, 2005.

9. OCHOA, E. R. et al. Marginal zone B-cell lymphoma of the salivary gland arising in chronic sclerosing sialadenitis (Kuttner tumor). Am J Surg Pathol, v. 25, n. 12, p. 1546-50, 2001.

10. SEIFERT, G.; SOBIN, H. Histological typing of salivary gland tumours. $2^{\text {nd }}$ ed. Berlin: Spring-Verlag, 1991. 